\section{Characteristics and Risk Factors for Candidemia in Newborns Admitted in Level III Neonatal Unit New Delhi}

A.Kumar*, A.Saili*, S.Namdev*, A.Mahor, ${ }^{* *}$, V.S.Randhawa**, S.Nangia*, From Departments of Neonatology* and Microbiology ${ }^{* *}$ Lady Hardinge Medical College and Associated Kalawati Saran Children

\section{Background}

Candida is one of the leading causes of bloodstream infections (BSI) in neonatal intensive care units (NICUs)

It has been estimated that $2.4-9.0 \%$ of mortality ${ }^{1,2}$ and $25.0 \%$ of morbidity in the NICU setting may be attributable to Candida infections ${ }^{3}$

Delay in recognition of Candida infections and in the initiation of appropriate antifungal therapy often leads to significant morbidity and mortality rates among high-risk infants ${ }^{4}$ Given the high mortality and difficulty in establishing an early diagnosis, it is important to determine risk factors for Candida BSI

\section{Objective}

To determine the proportion of Candida BSI admitted

To compare demographics of infants with invasive Candida BSI and matched controls

To find the risk factors for Candida BSI in newborns

\section{Methods}

Retrospective Case Control Study

Period between January 2014 and

December 2017 (48 months)

Level III NICU in New Delhi

Study population included babies who grew Candida on blood culture

Data on patient demographics, underlying diseases, medications, central catheters, nutrition, ventilator use etc. was retrieved.

Risk factors were evaluated from the time of admission until the onset of

\section{Hospital New Delhi
Heotology and Microbiology}

Candida BSI and then till discharge or death

For each case, one neonate with negative blood culture results was matched for admission age, gender, gestational age, and birth weight

Data analysis was performed using SPSS Version 20.0. Risk factors were assessed using univariate analysis Variables that were significant in univariate analysis or that have a priori clinical significance were entered into backward step wise regression model in multivariate analysis

All $p$ values were two tailed and values $<.05$ were taken as significant

\section{Risk Factors for Candida BSI}

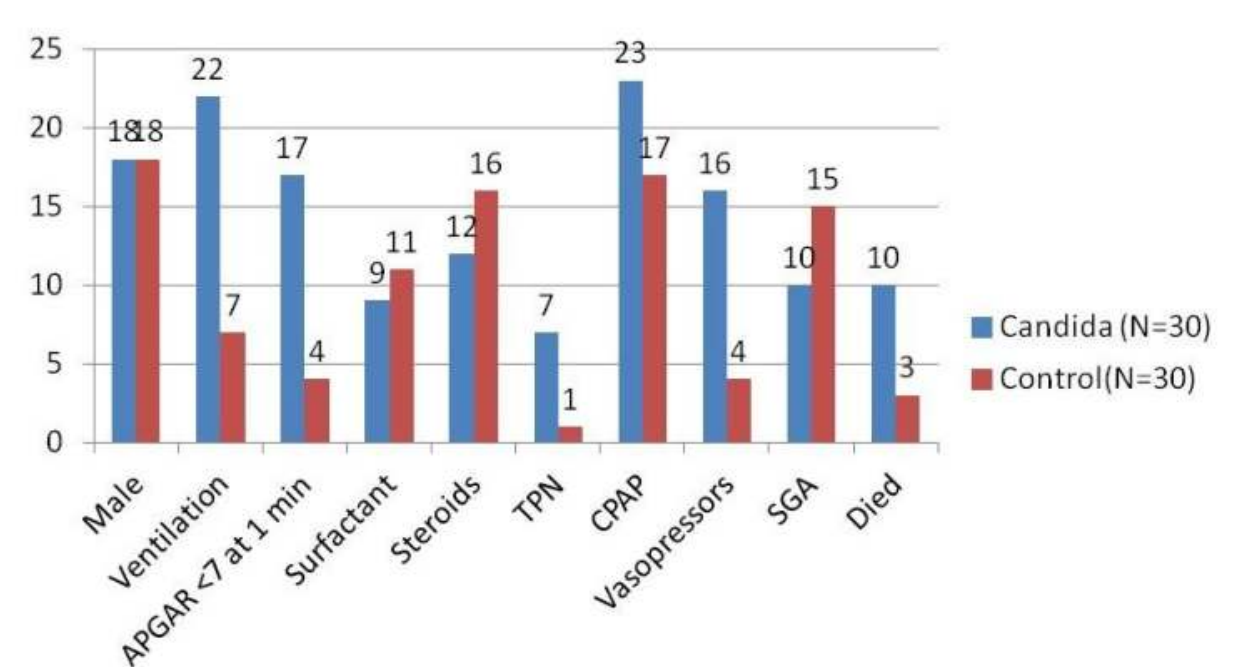

\section{Results}

Thirty out of total of 8928 admitted newborns developed Candida BSI (3.36 per 1000)

On univariate analysis factors associated with significantly higher risk of Candida BSI were Apgar score $<7$ at 1 min, Ventilation, Vasopressor use, TPN and Prolonged hospital stay

Apgar score $<7$ at $1 \mathrm{~min}$ and Vasopressor use were found to be

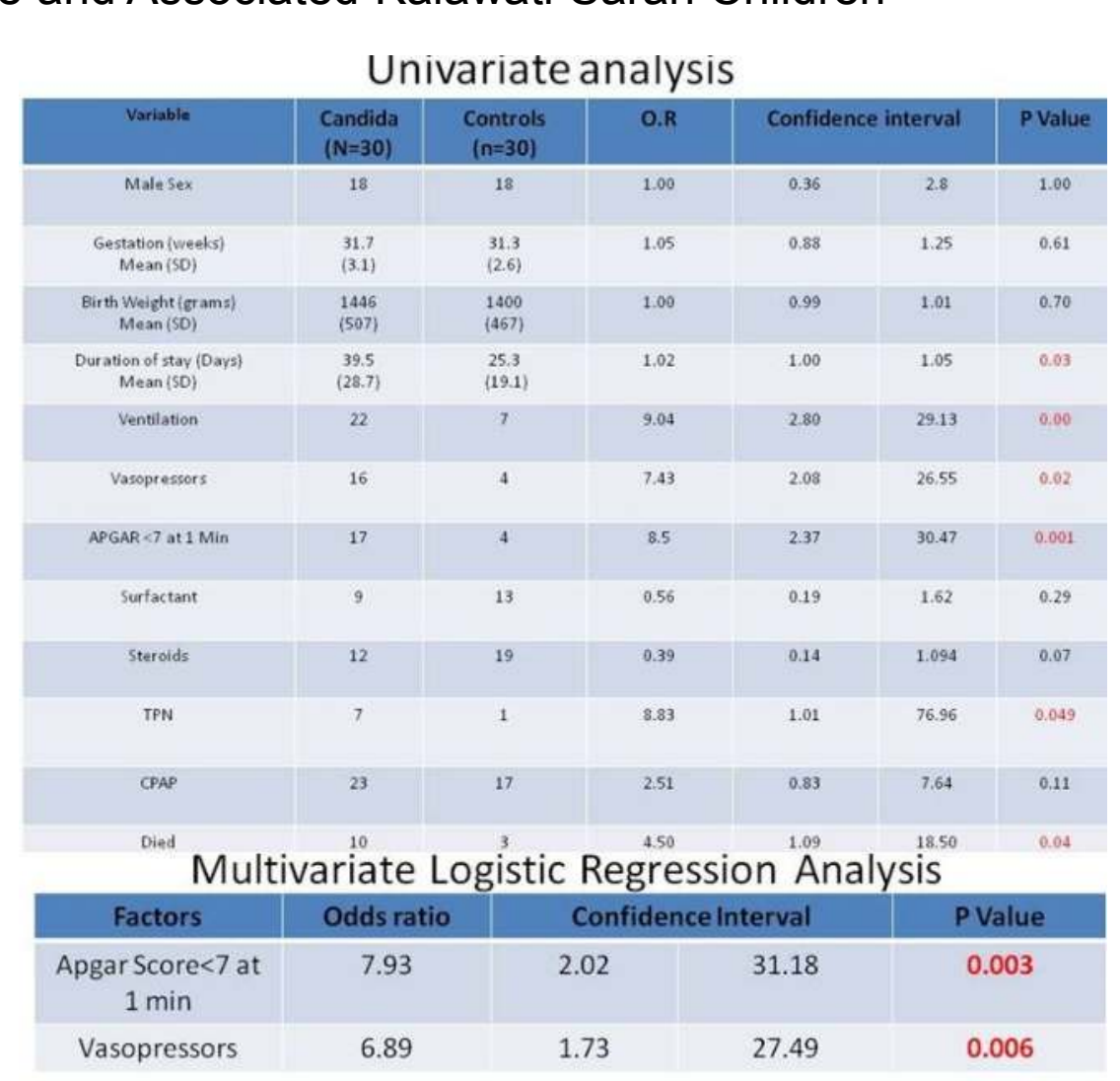

independent risk factors on multivariate analysis

Candida BSI was associated with significantly higher mortality $(33 \%$ vs. $10 \% p<0.05$ )

\section{Discussion}

The overall incidence of candidemia in our study was 3.36 per 1000 admissions. This incidence was lower than $13.6 / 1000^{4}$ and $9 \%{ }^{1}$ in a previous studies

In a study by Chen et $\mathrm{al}^{5}$ central venous catheterization and total parentera nutrition were identified as significant predisposing factors for candidemia. The multivariate logistic regression model results suggested that tota parenteral nutrition was the factor most highly associated with increased odds of candidemia. In our study factors associated with significantly higher risk of Candida BSI were apgar score $<7$ at 1 min, Ventilation, Vasopressor use, TPN and Prolonged hospital stay. However on multivaraite analysis Apgar score $<7$ at $1 \mathrm{~min}$ and Vasopressor use were found to be independent risk factors

The mortality of $33 \%$ reported in our study is comparable to $31 \%$ reported in an Indian study ${ }^{6}$ but higher than $5.8 \%$ reported from $\mathrm{China}^{7}$

\section{Limitations}

Retrospective Study

We were not able to assess all variables Limited by the completeness of documentation by the treating neonatologist

Study period and sample size was small We did not look for the colonization of the infants

Examination of environmental flora was not performed

Results could be NICU specific

\section{Conclusions}

Our study suggests that Apgar Score $<7$ at 1 minute and Vasopressor use are independent risk factors for Candidemia in Newborns.

Candida BSI was associated with significantly higher mortality.

\section{References}

1. Benjamin Jr DK, Stool BJ, Gantz MG, et al. Pediatrics 2010;126:865-73.

2. Oser $C$, Vergnano $S$, Naidoo $R$, et al.Clin Microbiol Infect 2014;20:936-41.

3. Benjamin Jr DK, Stoll BJ, Fanaroff AA. Pediatrics 2006;117:84-92.

4. Baley JE, Kliegman RM, Fanaroff AA. Pediatrics 1984;73:144-52.

5. Chen J, Jiang Y, Wei B, et al. BMC Infectious Diseases 2016;16: 700.

6. Wadile RG, Bhate VM. Indian J Pathol Microbiol 2015;58:472-4.

7. Fu J, Ding $Y$, Wei $B$, Wang $L$, et al. BMC Infectious Diseases 2017;17:329 